\title{
Weak values from path integrals
}

\author{
A. Matzkin \\ Laboratoire de Physique Théorique et Modélisation, CNRS Unité 8089, CY Cergy Paris Université, \\ 95302 Cergy-Pontoise cedex, France
}

(Received 6 February 2020; accepted 7 August 2020; published 24 August 2020)

\begin{abstract}
We connect the weak measurements framework to the path integral formulation of quantum mechanics. We show how Feynman propagators can in principle be experimentally inferred from weak value measurements. We also obtain expressions for weak values parsing unambiguously the quantum and the classical aspects of weak couplings between a system and a probe. These expressions are shown to be useful in quantum-chaos-related studies (an illustration involving quantum scars is given), and also in solving current weak-value-related controversies (we discuss the existence of discontinuous trajectories in interferometers and the issue of anomalous weak values in the classical limit).
\end{abstract}

DOI: 10.1103/PhysRevResearch.2.032048

There has been a growing interest in weak measurementsa specific form of quantum nondemolition measurementsover the past decade. Weak measurements were indeed found to be useful in fundamental or technical investigations, involving both experimental and theoretical works. Nevertheless, ever since their inception [1], weak values-the outcomes of weak measurements-have remained controversial. Since weak values are entirely derived from within the standard quantum formalism, the controversies have never concerned the validity of weak values (WV), but their understanding and their properties. For instance, are WV values similar to eigenvalues, or are they akin to expectation values [2,3]? Do anomalous WV represent a specific signature of quantum phenomena or can they be reproduced by classical conditional probabilities [4-7]? Are WV related to the measured system properties or do they represent arbitrary numbers characterizing the perturbation of the weakly coupled pointer $[8,9]$ ?

The path integral formulation of quantum mechanics is strictly equivalent to the standard formalism based on the Schrödinger equation. Though it is often technically more involved, path integrals give a conceptually clearer picture though the natural, built-in connection with quantities defined from classical mechanics (Lagrangian, action, paths). Surprisingly, very few works have employed weak values in a path integral context. Even then, the interest was restricted to the weak measurement of Feynman paths in semiclassical systems [10-12], to WV of specific operators [13-15], or as a way to probe virtual histories [16].

In this work, we connect the weak measurements framework to the path integral formulation. We will see that path integrals parse the quantum and classical aspects of weak values, thereby clarifying many of the current controversies involving weak measurements. In particular, we will show how

Published by the American Physical Society under the terms of the Creative Commons Attribution 4.0 International license. Further distribution of this work must maintain attribution to the author(s) and the published article's title, journal citation, and DOI. the path integral expression accounts for the discontinuous trajectories observed in interferometers and currently wildly debated [17-19]. We will also see that in the classical limit the weak value is washed out by coarse graining, implying that anomalous pointer values are a specific quantum feature with no classical equivalent [4-6]. A nice feature arising from the present approach is the possibility to measure propagators through weak measurements. This could be a useful tool in quantum-chaos-related studies as a method to observe the semiclassical amplitudes; we give an illustration in which the autocorrelation function employed to investigate quantum scars is inferred from weak values. The present approach could also be the starting point to apply weak values in domains, such as quantum cosmology, for which the standard, nonrelativistic weak measurements framework, relying on a von Neumann interaction, cannot be applied.

A weak measurement of a system observable $\hat{A}$ is characterized by four steps: (1) The system of interest is prepared in the chosen initial state $\left|\psi_{i}\right\rangle$, a step known as preselection. A quantum probe is prepared in a state $\left|\phi_{i}\right\rangle$, and the initial state is thus

$$
\left|\Psi\left(t_{i}\right)\right\rangle=\left|\psi_{i}\right\rangle\left|\phi_{i}\right\rangle .
$$

(2) The system and the probe are weakly coupled through an interaction Hamiltonian $\hat{H}_{\text {int }}$ (a so-called von Neumann interaction). (3) After the interaction, the system evolves until another system observable, say $\hat{B}$, is measured through a standard measurement process. Of all the possible eigenstates $\left|b_{k}\right\rangle$ that can be obtained, a filter selects only the cases for which $\left|b_{k}\right\rangle=\left|b_{f}\right\rangle$, where $\left|b_{f}\right\rangle$ is known as the postselected state. (4) When postselection is successful, the probe is measured. The final state of the probe $\left|\phi_{f}\right\rangle$ has changed relative to $\left|\phi_{i}\right\rangle$ by a shift depending on the weak value of $\hat{A}$.

Let $\hat{U}_{s}\left(t_{2}, t_{1}\right)$ denote the system evolution operator, and let $t_{i}, t_{w}$ and $t_{f}$ represent the preparation, interaction, and postselection times respectively. The weak value [1] of $\hat{A}$ is then given by

$$
A^{w}=\frac{\left\langle b_{f}\left(t_{f}\right)\left|\hat{U}_{s}\left(t_{f}, t_{w}\right) \hat{A} \hat{U}_{s}\left(t_{w}, t_{i}\right)\right| \psi\left(t_{i}\right)\right\rangle}{\left\langle b_{f}\left(t_{f}\right)\left|\hat{U}_{s}\left(t_{f}, t_{i}\right)\right| \psi\left(t_{i}\right)\right\rangle} .
$$


Typically, the probe state $\left|\phi_{i}\right\rangle$ is a Gaussian pointer initially centered at the position $Q_{w}$ and $\hat{H}_{\text {int }}$ is of the form

$$
\hat{H}_{\mathrm{int}}=g(t) \hat{A} \hat{P} f_{w},
$$

where $\hat{P}$ is the probe momentum, $g(t)$ is a function nonvanishing only in a small interval centered on $t_{w}$, and $f_{w}$ reflects the short-range character of the interaction that is only nonvanishing in a small region near $Q_{w}$. Under these conditions, it is well known [1] that $\left|\phi_{f}\right\rangle=e^{-i g A^{w} \hat{P}}\left|\phi_{i}\right\rangle$, with $g=\int d t^{\prime} g\left(t^{\prime}\right)$ and the final probe state is the initial Gaussian shifted by $g \operatorname{Re}\left(A^{w}\right)$.

In order to determine the evolution of the coupled systemprobe problem from the initial state $\left|\Psi_{i}\left(t_{i}\right)\right\rangle=\left|\psi_{i}\right\rangle\left|\phi_{i}\right\rangle$, we need the full Hamiltonian $\hat{H}=\hat{H}_{s}+\hat{H}_{p}+\hat{H}_{\text {int }}$ where $\hat{H}_{s}$ and $\hat{H}_{p}$ are the Hamiltonians for the uncoupled system and probe respectively. In terms of the corresponding evolution operators, the system and the probe evolve first independently, $\hat{U}_{0}\left(t, t_{i}\right)=\hat{U}_{s}\left(t, t_{i}\right) \hat{U}_{p}\left(t, t_{i}\right)$. Then, assuming for simplicity that the interaction takes place during the time interval $\left[t_{w}-\tau / 2, t_{w}+\tau / 2\right]$ ( $\tau$ is the duration of the interaction), the total evolution operator from $t_{i}$ to the postselection time $t_{f}$ is given as

$$
\begin{aligned}
\hat{U}\left(t_{f}, t_{i}\right)= & \hat{U}_{0}\left(t_{f}, t_{w}+\tau / 2\right) \hat{U}_{\mathrm{int}}\left(t_{w}+\tau / 2, t_{w}-\tau / 2\right) \\
& \times \hat{U}_{0}\left(t_{w}-\tau / 2, t_{i}\right) .
\end{aligned}
$$

The propagators $K \equiv\left\langle x_{2}\left|\hat{U}\left(t_{2}, t_{1}\right)\right| x_{1}\right\rangle$ for the uncoupled evolution of the system and probe are given respectively by

$$
\begin{array}{rl}
K_{s}\left(x_{2}, t_{2} ; x_{1}, t_{1}\right)=\int_{x_{1}}^{x_{2}} & \mathcal{D}[q(t)] \exp \left(\frac{i}{\hbar} \int_{t_{1}}^{t_{2}} L_{s}\left(q, \dot{q}, t^{\prime}\right) d t^{\prime}\right), \\
K_{p}\left(X_{2}, t_{2} ; X_{1}, t_{1}\right)= & \int_{X_{1}}^{X_{2}} \mathcal{D}[Q(t)] \\
& \times \exp \left(\frac{i}{\hbar} \int_{t_{1}}^{t_{2}} L_{p}(Q, \dot{Q}, t) d t^{\prime}\right)
\end{array}
$$

where as usual $[20,21] \mathcal{D}$ [.] implies integration over all paths connecting the initial and final space-time points, and $L_{s}=$ $\frac{m \dot{q}^{2}}{2}-V(q)$ and $L_{p}=\frac{M \dot{Q}^{2}}{2}$ are the classical system and probe Lagrangians respectively.

For the coupled evolution $\hat{U}_{\text {int }}$, the propagator becomes nonseparable,

$$
\begin{aligned}
K_{\text {int }} & \left(X_{2}, x_{2}, t_{2} ; X_{1}, x_{1}, t_{1}\right) \\
= & \int_{\left(X_{1}, x_{1}\right)}^{\left(X_{2}, x_{2}\right)} \mathcal{D}[Q(t)] \mathcal{D}[q(t)], \\
\quad & \times \exp \left[\frac{i}{\hbar} \int_{t_{1}}^{t_{2}} L\left(Q, \dot{Q}, q, \dot{q}, t^{\prime}\right) d t^{\prime}\right],
\end{aligned}
$$

where

$$
L=L_{s}+L_{p}-g(t) A(q) f\left(q, Q_{w}\right) M \dot{Q}
$$

is the classical interacting Lagrangian [22]; $f\left(q, Q_{w}\right)$ sets the range of the interaction (it becomes a Dirac $\delta$ function in the limit of a pointlike interaction). $Q_{w}$ will be taken here as a parameter specifying the position of the probe, which makes sense for a probe with a negligible kinetic term. Note that $A(q)$ gives the configuration space value of the classical dynamical variable $A(q)$.

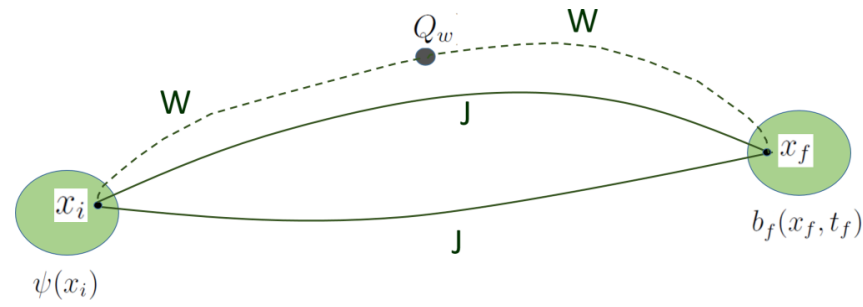

FIG. 1. Schematic illustration of the paths involved in the weak value expression when the pre- and postselected states are well localized, and the propagator is given as a sum over the classical trajectories [see Eq. (12)]. For the specific choice of postselection $b\left(x_{f}\right)=\delta\left(x-x_{f}\right)$, the weak value resulting from the coupling at $q=Q_{w}$ becomes proportional to the propagator $K_{s}\left(x_{f}, Q_{w}\right)$. The propagator can hence be observed from weak value measurements varying $Q_{w}$ and $x_{f}$.

Nonseparable propagators are notoriously difficult to handle except when they can be treated perturbatively [21], which is the case here. Standard path integral perturbation techniques (see Chap. 6 of Ref. [21]) applied to the system degrees of freedom give $K_{\text {int }}$ in terms of the uncoupled propagators and a first-order correction in which the system propagates as if it were uncoupled except that each path $q(t)$ is weighed by the perturbative term $g(t) A(q) f\left(q, Q_{w}\right) M \dot{Q}$. If the duration $\tau$ of the interaction is small relative to the other timescales (as is usually assumed in weak measurements), the timedependent coupling can be integrated to an effective coupling constant $g=\int_{t_{w}-\tau / 2}^{t_{w}+\tau / 2} g\left(t^{\prime}\right) d t^{\prime}$ and the uncoupled paths see an effective perturbation $g A(q) f\left(q, Q_{w}\right) M \dot{Q}$ at time $t_{w}$. Equation (7) becomes (the derivation is given in the Supplemental Material [22])

$$
\begin{aligned}
K_{\text {int }}= & K_{p} K_{s}+\int_{X_{1}}^{X_{2}} \mathcal{D}[Q(t)] e^{\frac{i}{\hbar} \int_{t_{1}}^{t_{2}} L_{p} d t^{\prime}} \int d q K_{s}\left(x_{2}, t_{2} ; q, t_{w}\right) \\
& \times A(q) f\left(q, Q_{w}\right) K_{s}\left(q, t_{w} ; x_{1}, t_{1}\right)\left(-\frac{i g}{\hbar} M \dot{Q}\right) .
\end{aligned}
$$

From the point of view of the system, the interpretation of Eq. (9) is straightforward (see Fig. 1): The transition amplitude from $x_{i}$ to $x_{f}$ involves a sum over the paths directly joining these two points in time $t_{f}-t_{i}$ as well as those that interact with the probe in the region determined by $f\left(q, Q_{w}\right)$, hence going from $x_{i}$ to some intermediate point $q$ within this region, and then from this point $q$ to $x_{f}$.

We now take into account the pre- and postselected states and focus on the probe evolution. For convenience, we perform the propagation from the initial state $\left|\Psi_{i}\right\rangle=\int d X_{i} d x_{i}\left|X_{i}\right\rangle\left|x_{i}\right\rangle \psi\left(x_{i}\right) \phi\left(X_{i}\right)$ up to the interaction time $t_{w}$. The postselected state $\left\langle b_{f}\right|=\int d x b_{f}^{*}\left(x_{f}\right)\left\langle x_{f}\right|$ is instead propagated backward to $t_{w}$. The uncoupled evolution $\left\langle b_{f}\left(t_{w}\right) \mid \psi_{i}\left(t_{w}\right)\right\rangle \phi_{i}\left(X, t_{f}\right)$ involves the direct paths from each $x_{i}$ where $\psi$ is nonvanishing to each $x_{f}$ lying in the support of $b_{f}(x)$. Factorizing this term in the full evolution, we get [22]

$$
\begin{aligned}
\phi\left(X, t_{f}\right) & =\left\langle b_{f}\left(t_{w}\right) \mid \psi_{i}\left(t_{w}\right)\right\rangle \\
& \times \int d X_{1} \int_{X_{1}}^{X} D[Q(t)] e^{\frac{i}{\hbar}\left[\int_{t_{w}}^{t_{f}} L_{p} d t^{\prime}-g A_{w} M \dot{Q}\right]} \phi_{i}\left(X_{1}, t_{w}\right),
\end{aligned}
$$


where $A^{w}$ is the weak value of $\hat{A}$ given by

$$
A^{w}=\frac{\int A(q) f\left(q, Q_{w}\right) K_{s}\left(x_{f}, t_{f} ; q, t_{w}\right) K_{s}\left(q, t_{w} ; x_{i}, t_{i}\right) b_{f}^{*}\left(x_{f}\right) \psi\left(x_{i}\right) d q d x_{f} d x_{i}}{\int d x_{f} d x_{i} K_{s}\left(x_{f}, t_{f} ; x_{i}, t_{i}\right) b_{f}^{*}\left(x_{f}\right) \psi\left(x_{i}\right)} .
$$

Several remarks are in order. First note that for large $M$ Eq. (10) implies a shift of the initial probe state, since the Lagrangian maps each point $X_{1}$ to $X_{1}-g A_{w}$. Second, while the denominator in Eq. (11) involves all the paths connecting the initial region to the final region, the numerator contains the sole paths connecting the initial and final regions passing through a point $q$ of the interaction region. Each such path is weighed by the classical value of the configuration space function $A(q)$ at that point. Note also that the weak value expressions (2) and (11) are both the results of asymptotic expansions, but the expansions are not exactly equivalent. For a contact interaction $f\left(q, Q_{w}\right)=\delta\left(q-Q_{w}\right)$, it is easy to see that Eqs. (2) and (11) become identical under the condition $A(q)=\langle q|\hat{A}| q\rangle$ for $q=Q_{w}$. In this case, $A^{w}$ is simply given by $A\left(Q_{w}\right) \times T_{w} / T$, where $T_{w}$ is the transition amplitude involving the paths connecting $x_{i}$ to $x_{f}$ by going through $Q_{w}$ at $t_{w}$, while $T$ connects $x_{i}$ to $x_{f}$ through any intermediate point) [see Fig. 1 and Eq. (12) below]. We then see the following: (i) The ratio $T_{w} / T$ can take any complex value; hence if $A\left(Q_{w}\right)$ is bounded, $\operatorname{Re}\left(A^{w}\right)$ can lie beyond these bounds and $A^{w}$ is said to be "anomalous." (ii) If the postselection state is chosen to be the space point $\left|x_{f}\right\rangle$, we have $A^{w}=A\left(Q_{w}\right) K_{s}\left(x_{f}, Q_{w}\right) \psi\left(Q_{w}, t_{w}\right) / \psi\left(x_{f}, t_{f}\right)$, so if the wave functions are known, e.g., through a previous weak-measurement-based procedure [23], the propagator $K_{s}\left(x_{f}, Q_{w}\right)$ can be obtained from the measurement of the weak value $A^{w}$. Both $x_{f}$ and $Q_{w}$ can be varied in order to measure the propagator over the region of interest.

Our weak value expression (11) is useful in cases involving free propagation or in the semiclassical approximation: For large actions, stationary phase integration transforms the sum over all arbitrary paths to a sum containing only the classical paths linking the initial and final points, so that both propagators (5) and (6) take the form $K^{s c}=$ $\sum_{k} \mathcal{A}_{k} \exp i\left[\mathcal{S}_{k} / \hbar-\pi \mu_{k} / 2\right]$, where $\mathcal{A}_{k}$ and $\mathcal{S}_{k}$ are the amplitude and classical action for the classical path $k$ connecting the initial and endpoints in time $t_{f}-t_{i}[20,24]$; the phase index $\mu_{k}$ counts the number of conjugate points along each trajectory and will be absorbed into the action to simplify the notation. The semiclassical regime, exact for free propagation, remains quantum since the different classical paths still interfere, and $A^{w}$ can be anomalous. Assuming again a pointlike interaction at $q=Q_{w}$, Eq. (11) becomes

$$
A^{w}=A\left(Q_{w}\right) \frac{\int d x_{f} d x_{i} \chi_{f}^{*}\left(x_{f}\right) \psi\left(x_{i}\right)\left[\sum_{W} \mathcal{A}_{W}\left(x_{f} ; Q_{w}\right) e^{i \mathcal{S}_{W}\left(x_{f} ; Q_{w}\right) / \hbar} \mathcal{A}_{W}\left(Q_{w} ; x_{i}\right) e^{i \mathcal{S}_{W}\left(Q_{w} ; x_{i}\right) / \hbar}\right]}{\int d x_{f} d x_{i} \chi_{f}^{*}\left(x_{f}\right) \psi\left(x_{i}, t_{i}\right) \sum_{J} \mathcal{A}_{J}\left(x_{f} ; x_{i}\right) e^{i \mathcal{S}_{J}\left(x_{f} ; x_{i}\right) / \hbar}} .
$$

$\mathcal{A}_{W}$ and $\mathcal{S}_{W}$ label the amplitude and action of a path going through $Q_{w}, W$ runs over all the classical paths connecting $x_{i}$ to $Q_{w}$ and $Q_{w}$ to $x_{f}$, while $J$ runs over all the classical paths connecting directly $x_{i}$ to $x_{f}$ in time $t_{f}-t_{i}$.

The form of the weak value given by Eq. (12) is particularly well suited to the experimental investigation of the contribution of individual orbits in semiclassical systems, such as the detection of quantum scars in wave functions that have recently received renewed interest [25]. The standard approach [26] to periodic orbit localization involves the autocorrelation function $\langle G(0) \mid G(t)\rangle$ where $G(x, 0)$ is a Gaussian with a maximum $x_{0}$ placed on a periodic orbit (po) that quickly spreads over all the available phase space. If the system is prepared and postselected in state $|G(0)\rangle$, the weak value of any observable $\hat{A}$ measured on a point $x_{p}$ of the periodic orbit is given by $A^{w}=\langle G(0)|$ $\hat{U}_{s}\left(t_{f}, t_{w}\right) \Pi_{x_{p}} \hat{A} \Pi_{x_{p}} \hat{U}_{s}\left(t_{w}, t_{i}\right)|G(0)\rangle /\left\langle G(0) \mid G\left(t_{f}\right)\right\rangle$, where $\Pi_{x_{p}}$ $\equiv\left|x_{x_{p}}\right\rangle\left\langle x_{x_{p}}\right|$ is the projector on $x_{p}$. By using Eq. (12), the autocorrelation function is obtained from the knowledge of the

\footnotetext{
${ }^{1}$ The standard definition of anomalous- $\operatorname{Re}\left(A^{w}\right)$ can lie above the largest or below the lowest eigenvalue-is more restrictive since by canonical quantization the eigenvalues range is typically contained within the possible values of $A(q)$ in configuration space.
}

(presumably observed) weak value and of a single Feynman path, the po:

$$
\begin{aligned}
\left\langle G(0) \mid G\left(t_{f}\right)\right\rangle= & A^{w}\left[\mathcal{A}_{p o}\left(x_{0}, x_{p}\right) A\left(x_{p}\right) \mathcal{A}_{p o}\left(x_{p}, x_{0}\right)\right. \\
& \left.\times e^{i S_{p o} / \hbar}\left|G\left(x_{0}, 0\right)\right|^{2}\right]^{-1} .
\end{aligned}
$$

Instead, the application of the standard weak value definition (2) to infer the autocorrelation function would require the knowledge of the full Schrödinger propagator.

Our approach is also relevant to understand current controversies involving WV such as the apparent observation of discontinuous trajectories [17-19] or the quantumness of anomalous weak values [4-6], as we briefly detail. The first of these issues involves a three-path interferometer depicted in Fig. 2. The weakly measured observable is the projector $\Pi_{j} \equiv\left|x_{j}\right\rangle\left\langle x_{j}\right|$, indicating whether the particle is at point $x_{j}$. By suitably choosing the preselected and postselected states, the following weak values are obtained [17]:

$$
\Pi_{A} \neq 0, \Pi_{E}=0, \Pi_{B} \neq 0, \Pi_{C} \neq 0, \Pi_{F}=0 .
$$

This means that weakly coupling a probe to the system leaves a trace on paths $A, B$, and $C$, but none on the segments $E$ and $F$ : The particle is seen inside the loop, but not on the entrance 


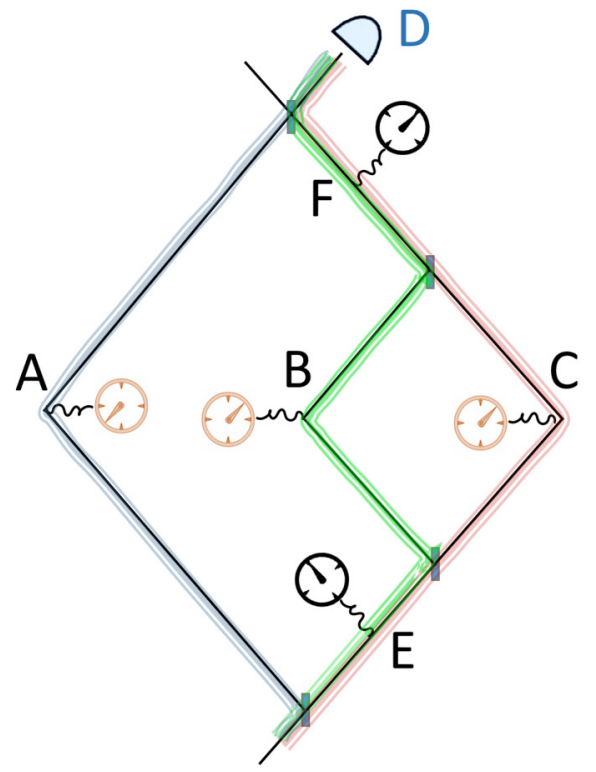

FIG. 2. A quantum particle propagating in an interferometer and postselected at $D$ displays discontinuous trajectories when observed with weak measurements [the weakly coupled probes depicted in black remain unaffected by the interaction with the particle, while the probes depicted in orange see their pointers shift after postselection; see Eq. (14)]. The Feynman paths of the particle, represented as pencils of trajectories along the arms, are continuous and propagate the interactions with the probes up to $D$, where superposition with the postselected state yield zero WV at $E$ and $F$ even when the wave function on these segments does not vanish (see text for details).

and exit paths. A point that has caused some confusion in the literature [18,19] is that in the initial proposal [17] the wave function vanished (by destructive interference) on paths $E$ and $F$, so that an interpretation in terms of vanishing weak values appeared to be flawed. It is possible, however, to enforce Eq. (14) without having destructive interference on $E$ and $F$. The present path-integral approach naturally parses the aspects relevant to a vanishing classical value, to a vanishing superposition, or to a partially propagated state being incompatible with postselection. Indeed, the WV expression (12) vanishes as follows:

(i) If $A\left(Q_{w}\right)=0$, which for a projector implies that the particle is not there;

(ii) if the term between brackets vanishes, corresponding to destructive interference of the wave function at $Q_{w}$ by summing over the different continuous paths $W$;

(iii) if the integral vanishes, that is the ensemble of points of the preselected state propagated by the sole paths going through $Q_{w}$ up to $x_{f}$ is orthogonal to the postselected state.

The case for asserting that the particle is not at $Q_{w}=E$ or $F$ when the WV vanishes is unproblematic in case (i): This is independent of postselection and would also be the case classically. Case (ii) corresponds to the initial proposal [17] in which two paths with opposite phases interfere destructively. This is a nonclassical effect [since we have $A\left(Q_{w}\right) \neq$ $0]$ but it does not necessarily depend on postselection and an interpretation in terms of weak values appears moot: If there is no wave function, there is nothing to measure, as remarked in Ref. [19]. The genuinely interesting case is case (iii): The paths do not interfere destructively in the interaction region, but the fraction of the preselected wave function propagated by the paths passing through this region (here $E$ or $F$ ) is incompatible with the postselected state. In other interaction regions (here $B$ or $C$ ), the paths contributing to the propagator in Eq. (12) are not orthogonal to the postselected state anymore. The crucial point is that the paths contained in the propagator are continuous, but each probe's motion results from the overlap at $t=t_{f}$ between the preselected state propagated by the paths hitting the probe region and the postselected state.

The other current controversy mentioned above concerns the quantumness of anomalous weak values that has been recently questioned as resulting from a mere statistical effect [4-7]. A path integral approach is convenient to obtain heuristically the classical limit of the weakly coupled probe's motion. This involves two well-known steps. The first step, deriving the semiclassical propagator $K^{s c}$, was recalled above. The second one involves retaining only the diagonal terms of the semiclassically propagated density matrix [27], due to coarse graining over a classical scale [28]. We then obtain [22] classical amplitudes obeying the classical transport equation, and the weak probe's dynamics is given by solving for each point of the initial distribution the equations of motion for the Lagrangian given by Eq. (8). Postselection is specified in terms of a domain $\mathfrak{B}_{f}$ at time $t_{f}$ defined such that the chosen configuration space function $b\left(q, t_{f}\right) \in \mathfrak{B}_{f}$. The average probe shift with postselection is then

$$
\langle\Delta Q\rangle=g \int_{\mathfrak{B}_{w}} A\left(q, t_{w}\right) f\left(q, Q_{w}\right) \frac{\rho_{s}\left(q, t_{w}\right)}{\int_{\mathfrak{B}_{w}} \rho_{s}\left(q^{\prime}, t_{w}\right) d q^{\prime}} d q .
$$

The integral is taken at $t_{w}$ over the configuration space domain $\mathfrak{B}_{w}$ such that at $t_{f}$ the condition $b\left(q, t_{f}\right) \in \mathfrak{B}_{f}$ is obeyed. $\langle\Delta Q\rangle$ can never be anomalous. Classically, the only way to have an anomalous probe shift would be to replace $A\left(q, t_{w}\right)$ by a different configuration space function or to have the numerator and denominator integrated over different domains, say, $\mathfrak{B}_{f}$ and $\mathfrak{B}_{f}^{\prime}$. In both cases, this would be the result of a perturbation, due to the detection process in the latter case.

To sum up, we have obtained an expression of weak values from a path integral approach. We have shown this expression to be useful when semiclassical propagators are involved. We have seen that the present approach gives a consistent account of two current controversies involving weak values (the observation of discontinuous trajectories and the quantumness of anomalous weak values). Other recent perplexing results [29] involving pre- and postselected ensembles can be treated similarly. We have also suggested a method for measuring the Feynman propagator through weak values. The approach introduced in this work will be fruitful to tackle extensions of the weak measurements framework to relativistic and cosmological settings, where quantities analogous to weak values are known to emerge [30]. 
[1] Y. Aharonov, D. Z. Albert, and L. Vaidman, Phys. Rev. Lett. 60, 1351 (1988).

[2] L. Vaidman, A. Ben-Israel, J. Dziewior, L. Knips, M. Weiß1, J. Meinecke, C. Schwemmer, R. Ber, and H. Weinfurter, Phys. Rev. A 96, 032114 (2017).

[3] J. Dressel, M. Malik, F. M. Miatto, A. N. Jordan, and R. W. Boyd, Rev. Mod. Phys. 86, 307 (2014).

[4] C. Ferrie and J. Combes, Phys. Rev. Lett. 113, 120404 (2014).

[5] M. F. Pusey, Phys. Rev. Lett. 113, 200401 (2014).

[6] J. Dressel, Phys. Rev. A 91, 032116 (2015).

[7] A. Romito, A. N. Jordan, Y. Aharonov, and Y. Gefen, Quant. Stud. Math. Found. 3, 1 (2016).

[8] A. Matzkin, Found. Phys. 49, 298 (2019).

[9] D. Sokolovski, Phys. Lett. A 379, 1097 (2015).

[10] A. Matzkin, Phys. Rev. Lett. 109, 150407 (2012).

[11] T. Mori and I. Tsutsui, Prog. Theor. Exp. Phys. 2015, 043A01 (2015).

[12] L. P. Withers, Jr. and F. A. Narducci, J. Math. Phys. 56, 062106 (2015).

[13] A. Tanaka, Phys. Lett. A 297, 307 (2002).

[14] R. Flack and B. J. Hiley, Entropy 20, 367 (2018).

[15] D. Sokolovski, Phys. Rev. A 96, 022120 (2017).

[16] D. Georgiev and E. Cohen, Phys. Rev. A 97, 052102 (2018).

[17] A. Danan, D. Farfurnik, S. Bar-Ad, and L. Vaidman, Phys. Rev. Lett. 111, 240402 (2013).

[18] L. Vaidman, Phys. Rev. A 89, 024102 (2014); P. L. Saldanha, ibid. 89, 033825 (2014); V. Potoček and G. Ferenczi, ibid. 92, 023829 (2015); R. B. Griffiths, ibid. 94, 032115 (2016); F. A. Hashmi, F. Li, S.-Y. Zhu, and M. S. Zubairy, J. Phys. A 49, 345302 (2016); Q. Duprey and A. Matzkin, Phys. Rev. A 95, 032110 (2017); D. Sokolovski, Phys. Lett. A 381, 227 (2017); Q. Z. Yuan and X. Feng, Phys. Rev. A 99, 053805 (2019).
[19] K. Bartkiewicz, A. Černoch, D. Javurek, K. Lemr, J. Soubusta, and J. Svozilik, Phys. Rev. A 91, 012103 (2015); B. G. Englert, K. Horia, J. Dai, Y. L. Len, and H. K. Ng, ibid. 96, 022126 (2017).

[20] L. S. Schulman, Techniques and Applications of Path Integration (Dover, New York, 2005).

[21] R. P. Feynman and A. R. Hibbs, Quantum Mechanics and Path Integrals (Dover, New York, 2010).

[22] See Supplemental Material at http://link.aps.org/supplemental/ 10.1103/PhysRevResearch.2.032048 for more details concerning the computation of the path integral with the interacting Lagrangian, and for the approach employed to obtain the classical limit.

[23] J. S. Lundeen et al., Nature (London) 474, 188 (2011).

[24] M. Brack and R. Bhaduri, Semiclassical Physics (AddisonWesley, Reading, MA, 2003).

[25] L. Huang, Y.-C. Lai, D. K. Ferry, S. M. Goodnick, and R. Akis, Phys. Rev. Lett. 103, 054101 (2009); M.-Y. Song, Z.-Y. Li, H.-Y. Xu, L. Huang, and Y.-C. Lai, Phys. Rev. Research 1, 033008 (2019); J. Keski-Rahkonen, A. Ruhanen, E. J. Heller, and E. Räsänen, Phys. Rev. Lett. 123, 214101 (2019).

[26] E. J. Heller, Phys. Rev. Lett. 53, 1515 (1984).

[27] P. Ajanapon, Am. J. Phys. 55, 159 (1986); B. Bhatt, M. R. Chander, R. Patil, R. Mishra, S. Nahar, and T. P. Singh, Zeit. Nat. A 75, 131 (2020).

[28] N. Wiebe and L. E. Ballentine, Phys. Rev. A 72, 022109 (2005); J. Kofler and C. Brukner, Phys. Rev. Lett. 99, 180403 (2007); A. Matzkin, Phys. Rev. A 84, 022111 (2011); H. Jeong, Y. Lim, and M. S. Kim, Phys. Rev. Lett. 112, 010402 (2014); R. G. Littlejohn, J. Stat. Phys. 68, 7 (1992).

[29] Y. Aharonov et al., PNAS 114, 6480 (2017).

[30] F. Englert and P. Spindel, J. High Energy Phys. 12 (2010) 065; E. Cohen and M. Nowakowski, Phys. Rev. D 97, 088501 (2018). 